\title{
Comunicação
}

\section{Padrão de sensibilidade de 117 amostras clínicas de Staphylococcus aureus isoladas em 12 hospitais}

\author{
W.V.L. Farias, H.S. SAder, I.L. Leme, A.C. Pignatari \\ Laboratório Especial de Microbiologia Clínica, Disciplina de Doenças I nfecciosas e Parasitárias, Universidade F ederal de São Paulo — \\ Escola Paulista de Medicina, São Paulo, SP.
}

RESUMO - OвJ etıvo. Avaliar o padrão de sensibilidade in vitro de amostras clínicas de Staphylococcus aureus sensíveis (OSSA) e resistentes à oxacilina (ORSA) a outros antimicrobianos que podem ser utilizados no tratamento de infecções estafilocócicas.

Material e Método. Foram analisadas 117 amostras clínicas de S. aureus isoladas em vários hospitais de São Paulo. Também foram incluídas amostras isoladas em Campinas, SP, e J oão Pessoa, PB. A avaliação da sensibilidade in vitro aos antimicrobianos foi realizada pela técnica de microdiluição em caldo, utilizando os procedimentos preconizados pelo National Committee for Clinical Laboratory Standards (NCCLS). Foi avaliada a concentração inibitória mínima (MIC) para 24 antimicrobianos da classe dos $\beta$-lactâmi cos, fluoroquinolonas, aminoglicosídeos, glicopeptídeos, macrolídeos, lincosaminas e estreptograminas. Foram avaliadas tanto drogas disponíveis comercialmente quanto as que ainda se encontram em fase de pesquisa. A resistência cruzada entre dez fluoroquinolonas foi avaliada em 24 amostras.

Resultados. Os glicopeptídeos, O RP-59500 e a mupirocina foram os antimicrobianos que apre-

\section{INTRODUÇÃO}

As infecções causadas por Staphylococcus aureus, tanto hospitalares quanto domiciliares, apresentam morbidade e mortalidade el evadas. $\mathrm{O}$ aumento crescente da freqüência de $S$. aureus resistentes a oxacilina (ORSA) e a possibilidade do aparecimento de amostras resistentes a vancomicina tornam importante o desenvolvimento de novas drogas com atividade antiestafilocócicas.

O primeiro grande surto de ORSA descrito na literatura ocorreu em 1963'; desde essa data, as publicações mostrando surtos intra-hospitalares causados por essa bactéria tornaram-se cada vez mais freqüentes, assumindo proporções assustadoras. A ocorrência de casos na comunidade também vem se tornando um problema, principalmente em usuários de drogas intravenosas. Amostras sentaram maior atividade in vitro contra amostras de ORSA ( $100 \%$ sensibilidade). Oitenta e sete por cento das amostras de ossA foram sensíveis à ciprofloxacina ( $\mathrm{MIC}_{50} 0,25 \mu \mathrm{g} / \mathrm{mL}$ ), enquanto que, para os ORSA, a sensibilidade foi de apenas $38 \%$ $\left(\mathrm{MIC}_{50}>4 \mu \mathrm{g} / \mathrm{mL}\right.$ ). A resistência cruzada para as fluoroquinolonas foi observada mesmo para drogas não disponíveis comercialmente. As fluoroquinolonas que permaneceram ativas contra amostras resistentes à ciprofloxacina (clinafloxacina e wiN-57.273) apresentaram MICs 8 a 64 vezes mais elevados que as amostras sensíveis à ciprofloxacina, sugerindo que, quando lançadas na prática clínica, esses mics possam se elevar ainda mais, inviabilizando o uso clínico desses compostos.

Conclusão. Os resultados do presente estudo mostraram uma alta taxa de resistência a antimicrobianos das amostras de $S$. aureus nos hospitais do Brasil, restando poucas opções para o tratamento de infecções causadas por ORSA.

UNITERMOS: Staphylococcus aureus. Atividade antimicrobiana in vitro. Resistência a antimicrobianos. Quinolonas. Glicopeptídeos.

de ORSA são, geralmente, resistentes a inúmeros antimicrobianos, incluindo todos os outros $\beta$-lactâmicos, os macrolídeos, as lincosaminas, os aminoglicosídeos, o cloranfenicol e a tetraciclina.

A vancomicina é o antibiótico de escol ha para o tratamento de infecções causadas por ORSA ${ }^{1}$. Apesar de ainda não terem sido descritas amostras de S. aureus resistentes aos glicopeptídeos, existem relatos de resistência relativa (MIC $=8 \mu \mathrm{g} / \mathrm{mL}$, o qual é interpretado como intermediário pelo $\mathrm{Na}$ tional Committee for Clinical Laboratory Standards [NCCLS]) a essa classe de anti mi crobianos em estafilococos coagulase negativos $(E C N)^{2}$, especialmente S. haemolyticus ${ }^{3}$, e em espécies de enterococos ${ }^{4}$. A evidência de resistência aos glicopeptí deos mediada por plasmídeo em enterococos é um motivo de grande preocupação por causa do risco potencial de disseminação dessa resistência 
para outras espécies de bactérias gram-positivas, principalmente S. aureus. Em recente trabalho, Daum et al..$^{5}$ observaram uma diminuição da sensibilidade de $\mathrm{S}$. aureus, tanto a vancomicina quanto a teicoplanina, quando essas bactérias eram incubadas de forma seriada em baixas concentrações de vancomicina. Isso aumenta ainda mais a preocupação com o surgimento de amostras de S. aureus resistentes aos glicopeptídeos.

Os laboratórios de microbiologia desempenham papel importante não somente na detecção de resistência aos antimicrobianos de uso clínico como, também, às drogas que possam, futuramente, ser utilizados no tratamento de infecções causadas por amostras de S. aureus multirresistentes. Em virtude da facilidade que essa espéci e tem demonstrado para desenvolver resistência a antimicrobianos, é provável que tenhamos dificuldade no tratamento de infecções estafilocócicas. O objetivo do presente estudo foi avaliar novas opções terapêuticas para o tratamento de infecções causadas por S. aureus isolados nos hospitais brasileiros, em particular os de São Paulo.

\section{MATERIAIS E MÉTODO}

Foram avaliadas 117 amostras clínicas de S. aureus isoladas em 11 hospitais, no período de setembro de 1990 a janeiro de 1992. As amostras foram retiradas do banco de microrganismos do laboratório especial de microbiologia clínica (LEMC) da Disciplina de Doenças I nfecciosas e Parasitárias da Escola Paulista de Medicina (UNIFESP). Os hospitais que forneceram as amostras foram os seguintes: Hospital São Paulo (21 amostras), Hospital Eval do F oz (4 amostras), Hospital Brigadeiro (4 amostras), Hospital Nove deJ ulho (5 amostras), Hospital Oswaldo Cruz (17 amostras), Hospital do Servidor Público Estadual de São Paulo (7 amostras), Hospital Humberto Primo (25 amostras), Hospital Sírio Libanês (11 amostras), Hospital Dante Pazzanese (5 amostras), Hospital das Clínicas da Universidade de Campinas (UNICAMP, 5 amostras), Hospital das Clínicas da Universidade F ederal da Paraíba (UFPb, 13 amostras).

F oram utilizados os métodos convencionais para identificação de gênero e espécie, tais como coloração de Gram, prova de coagulase, staph-test e prova de crescimento em ágar contendo manitol e $\mathrm{NaCl}$ a $6 \%$. Em uma etapa posterior, as amostras foram enviadas para o laboratório especial de microbiologia (Special Microbiology Laboratory) da Universidade de lowa, em lowa City, EUA, onde foram realizados os demais procedimentos laboratoriais. Os testes de sensibilidade aos antimicro- bianos foram realizados pela técnica de microdiluição em caldo, utilizando os procedimentos padronizados pelo NCCLS ${ }^{6}$. Os antimicrobianos utilizados na preparação das placas foram fornecidos pelas companhias responsáveis pela comercialização das respectivas drogas. Os antimicrobianos testados incluíam drogas das classes dos $\beta$ lactâmicos (meticilina, oxacilina e penicilina), fluoroquinol onas (ciprofloxacina), aminoglicosídeos (amicacina, tobramicina e gentamicina), glicopeptídeos (vancomicina, teicoplanina, ramoplanina, daptomicina, MDL-628737 e LY-2648267), macrolídeos (eritromicina, azitromicina, claritromicina, roxitromicina), lincosaminas (clindamicina), estreptograminas (RP-59.500 $)$, além de outras drogas com ação antiestafilocócica, tais como cloranfenicol, mupirocina, rifampicina, tetraciclina e trimetoprim.

A resistência cruzada entre drogas da classe das fluoroquinolonas foi avaliada através do teste de 24 amostras (19 resistentes a ciprofloxacina e 5 sensíveis a este antimicrobiano) para as seguintes drogas: ciprofloxacina, ofloxacina, fleroxacina, lomefloxacina, clinafloxacina, temafloxacina, grepafloxacina (OPC-17116), E-4695, E-4868 e WIN572737. As fluoroquinolonas também foram avaliadas pelo método de microdiluição em caldo ${ }^{6}$.

\section{RESULTADOS E DISCUSSÃO}

Das 117 amostras avaliadas, 38 foram sensíveis à oxacilina (OSSA, MIC $\leq 2 \mu \mathrm{g} / \mathrm{mL})^{6}$ e 79 resistentes (ORSA, MIC $\geq 4 \mu \mathrm{g} / \mathrm{mL}$ ) 6 . É importante lembrar que as amostras não for am col etadas consecutivamente ou até no mesmo período nos diferentes hospitais. Dessa maneira, não é possível tirar nenhuma conclusão com relação a prevalência de resistência a antimicrobianos nos hospitais estudados ou mesmo na região onde foi feito o estudo. O objetivo do estudo foi avaliar novas opções terapêuticas para o tratamento de infecções estafilocóci cas e comparar o perfil de sensibilidade de amostras sensíveis à oxacilina com amostras resistentes.

As tabelas 1 e 2 mostram a sensibilidade de amostras de ORSA e de OSSA a antimicrobianos de diferentes classes. Antimicrobianos da classe dos macrolídeos apresentaram atividade baixa, tanto para OSSA quanto para ORSA. A baixa sensibilidade de amostras de OSSA aos macrolídeos foi inesperada, visto que dados de literatura internacional sugerem que, aproximadamente, $80 \%$ dessas cepas sejam sensíveis a essa classe de antimi crobianos $^{9}$. Por outro lado, outros trabal hos também têm mostrado a resistência de amostras de ORSA a essa mesma classe ${ }^{10}$. 


\begin{tabular}{|c|c|c|c|c|}
\hline $\begin{array}{l}\text { Antimicrobiano } \\
\beta \text {-lactâmicos }\end{array}$ & $\mathrm{MIC}_{50}(\mu \mathrm{g} / \mathrm{mL})$ & $\mathrm{MIC}_{90}(\mu \mathrm{g} / \mathrm{mL})$ & Variação & $\%$ Sensibilidade \\
\hline Penicilinas & $>1$ & $>1$ & $\leq 0,03->1$ & 18 \\
\hline Oxacilina & $\leq 0,25$ & 1 & $\leq 0,25-2$ & 100 \\
\hline Meticilina & 2 & 4 & $\leq 1-8$ & 100 \\
\hline \multicolumn{5}{|l|}{ Aminoglicosídeos } \\
\hline Amicacina & $\leq 2$ & 32 & $\leq 2->64$ & 84 \\
\hline Gentamicina & $\leq 0,5$ & $>16$ & $\leq 0,5->16$ & 84 \\
\hline Tobramicina & $\leq 0,5$ & $>16$ & $\leq 0,5->16$ & 76 \\
\hline Clindamicina & $\leq 0,25$ & $>8$ & $\leq 0,25->8$ & 84 \\
\hline Cloranfenicol & 8 & 16 & $4->32$ & 87 \\
\hline Rifampicina & $\leq 0,12$ & 2 & $\leq 0,12->4$ & 84 \\
\hline Tetraciclina & $\leq 0,5$ & $>32$ & $\leq 0,5->16$ & 58 \\
\hline Trimetoprim & 2 & $>8$ & $0,5->8$ & 71 \\
\hline \multicolumn{5}{|l|}{ Macrolídeos } \\
\hline Eritromicina & $>16$ & $>16$ & $\leq 0,25->16$ & 45 \\
\hline Azitromicina & 4 & $>16$ & $\leq 0,12->16$ & 47 \\
\hline Claritromicina & 0,5 & $>16$ & $\leq 0,12->16$ & 58 \\
\hline Roxitromicina & 8 & $>16$ & $\leq 0,12->16$ & 45 \\
\hline Mupirocina & $\leq 0,5$ & $\leq 0,5$ & $\leq 0,5$ & 100 \\
\hline \multicolumn{5}{|l|}{ Fluoroquinolona } \\
\hline Ciprofloxacina & 0,25 & 2 & $\leq 0,12->4$ & 87 \\
\hline \multicolumn{5}{|l|}{ Estreptogramina } \\
\hline RP 59500 & 0,5 & 0,5 & $0,12-1$ & 100 \\
\hline \multicolumn{5}{|l|}{ Glicopeptídeos } \\
\hline Vancomicina & 0,5 & 1 & $0,5-1$ & 100 \\
\hline Teicoplanina & 0,5 & 1 & $0,25-4$ & 100 \\
\hline Daptomicina & 2 & 4 & $\leq 0,12-4$ & 100 \\
\hline Ramoplanina & 1 & 1 & $\leq 0,5-2$ & 100 \\
\hline MDL - 62873 & $\leq 0,12$ & 0,25 & $0,12-1$ & 100 \\
\hline LY - 264826 & $\leq 0,12$ & 0,25 & $0,12-0,25$ & 100 \\
\hline
\end{tabular}

Outra classe de antimicrobianos estudada foi a dos aminoglicosídeos. A maioria das amostras de OSSA avaliadas foram sensíveis a antimicrobianos dessa classe. Mais uma vez, foi notada diferença importante entre o padrão de sensibilidade de amostras de OSSA e de ORSA, com apenas uma pequena percentagem das amostras de ORSA sendo sensíveis aos aminoglicosídeos testados. A avaliação do padrão de sensibilidade de $\mathrm{S}$. aureus aos aminoglicosídeos se faz importante, uma vez que essa classe de antimicrobianos pode ser utilizada em associação com $\beta$-lactâmi cos ou gl icopeptídeos, para o tratamento de infecções graves.

$\mathrm{O}$ padrão de sensibilidade de $\mathrm{S}$. aureus, especialmente ORSA, ao trimetoprim pode variar bastante de uma instituição para outra. Enquanto al guns estudos apresentam alta sensibilidade das amostras de ORSA ${ }^{1}$, outros trabal hos mostram que esse patógeno tende a ser resistente também a trimetoprim. Essa diferença regional pode estar relacionada ao uso do antimi crobiano. N o presente estudo, apenas $18 \%$ das amostras de ORSA ( MIC $_{50}>8 \mu \mathrm{g} /$ $\mathrm{mL}$ ) e $71 \%$ das amostras de OSSA ( $\mathrm{MIC}_{50} 2 \mu \mathrm{g} / \mathrm{mL}$ ) foram sensíveis a esse antimicrobiano.

Todas as amostras estudadas foram sensíveis à vancomicina $\left(\mathrm{MIC}_{90} 1 \mu \mathrm{g} / \mathrm{mL}\right)$, à teicoplanina $\left(\mathrm{MIC}_{90} 1 \mu \mathrm{g} / \mathrm{mL}\right.$ ) e aos demais gl icopeptídeos testados (ramoplanina, daptomicina, MDL-62873 e LY264826). É importante lembrar que os estudos com a daptomicina foram suspensos por causa da toxicidade que esse composto apresentou em estudos in vivo. Além disso, nenhum dos outros glicopeptídeos avaliados se encontra disponível comercialmente no Brasil ou mesmo em outros países.

O RP-59.500, uma nova estreptogramina que tem demonstrado excel ente atividade in vitro contra amostras de enterococos resistentes à vancomicina ${ }^{8}$, também apresentou excelente atividade contra amostras dos dois grupos ( $100 \%$ de sensibilidade, $\mathrm{MIC}_{90} 0,5$ e $1 \mu \mathrm{g} / \mathrm{mL}$ para OSSA e ORSA, respectivamente). Mais estudos clínicos são necessários para avaliar o papel dessa droga na terapêutica antiestafilocócica.

No presente estudo, não foi detectada nenhuma amostra resistente à mupirocina $\left(\mathrm{MIC}_{90} \leq 0,5 \mu \mathrm{g} /\right.$ $\mathrm{mL}$ ), porém resistência a esse antimicrobiano em $\mathrm{S}$. aureus tem sido relatada em centros que empregaram essa droga em programas de descol onização nasal de ORSA ${ }^{11,12}$. Provavelmente, o fato de essa droga não ser disponível nos hospitais na época do 


\begin{tabular}{|c|c|c|c|c|}
\hline $\begin{array}{l}\text { Antimicrobiano } \\
\beta \text {-lactâmicos }\end{array}$ & \multicolumn{3}{|c|}{$\beta$-lactâmicos } & $\%$ Sensibilidade \\
\hline Penicilinas & $>1$ & $>1$ & $>1$ & 0 \\
\hline Oxacilina & $>8$ & $>8$ & $4->8$ & 0 \\
\hline Meticilina & $>32$ & $>32$ & $16->32$ & 0 \\
\hline \multicolumn{5}{|l|}{ Aminoglicosídeos } \\
\hline Amicacina & 64 & $>64$ & $\leq 2->64$ & 9 \\
\hline Gentamicina & $>16$ & $>16$ & $\leq 0,5->16$ & 4 \\
\hline Tobramicina & $>16$ & $>16$ & $1->16$ & 3 \\
\hline \multicolumn{5}{|l|}{ Macrolídeos } \\
\hline Eritromicina & $>8$ & $>8$ & $\leq 0,25->8$ & 4 \\
\hline Azitromicina & $>16$ & $>16$ & $\leq 0,12->16$ & 4 \\
\hline Claritromicina & $>16$ & $>16$ & $\leq 0,12->16$ & 5 \\
\hline Roxitromicina & $>16$ & $>16$ & $\leq 0,12->16$ & 4 \\
\hline Clindamicina & $>8$ & $>8$ & $\leq 0,25->8$ & 6 \\
\hline Cloranfenicol & $>32$ & $>32$ & $4->32$ & 13 \\
\hline Rifampicina & 2 & $>4$ & $\leq 0,12->4$ & 15 \\
\hline Tetraciclina & $>16$ & $>16$ & $\leq 0,5->16$ & 10 \\
\hline Trimetoprim & $>8$ & $>8$ & $\leq 0,25->8$ & 18 \\
\hline \multicolumn{5}{|l|}{ Fluoroquinolona } \\
\hline Ciprofloxacina & $>4$ & $>4$ & $\leq 0,12->4$ & 38 \\
\hline Mupirocina & $\leq 0,5$ & $\leq 0,5$ & $\leq 0,5-1$ & 100 \\
\hline \multicolumn{5}{|l|}{ Estreptogramina } \\
\hline RP59500 & 0,5 & 1 & $\leq 0,25-1$ & 100 \\
\hline \multicolumn{5}{|l|}{ Glicopeptídeos } \\
\hline Vancomicina & 0,5 & 1 & $\leq 0,25-2$ & 100 \\
\hline Teicoplanina & 1 & 2 & $0,5-4$ & 100 \\
\hline Daptomicina & 4 & 4 & $2-8$ & 100 \\
\hline Ramoplanina & 1 & 2 & $1-2$ & 100 \\
\hline MDL - 62873 & $\leq 0,12$ & 0,25 & $\leq 0,12-0,25$ & 100 \\
\hline LY - 264826 & $\leq 0,12$ & $\leq 0,12$ & $\leq 0,12-0,5$ & 100 \\
\hline
\end{tabular}

estudo, provavelmente, foi responsável pelo fato de não ter si do i solada amostra resistente à mupirocina. Após a introdução dessa droga na prática clínica, talvez comecem a se observar casos de infecções causadas por S. aureus resistentes.

As fluoroquinolonas também foram testadas, sendo a ciprofloxacina a representante dessa classe. Oitenta e sete por cento das amostras de OSSA foram sensíveis à ciprofloxacina. Porém, a ciprofloxacina mostrou baixa atividade contra amostras de ORSA, com somente $38 \%$ das amostras sendo sensíveis. Cada vez mais surgem publicações que mostram a aquisição de resistência por parte desse microrganismo às fluoroquinol onas ${ }^{13}$. São vários os mecanismos pelos quais o $\mathrm{S}$. aureus desenvolve resistência às fluoroquinol onas. O mecanismo mais importante e mais freqüente é decorrente de al teração no sítio de ação desses antimicrobianos (DNA girase) e se deve a mutações no gene Gyr $A^{14}$. Também é descrita resistência mediada por transporte ativo da droga para fora da bactéria ${ }^{14}$.

A avaliação de resistência cruzada entre ciprofloxacina e outros representantes das fluoroquinoIonas foi avaliada em um número menor de amostras (tabela 3). Alguns trabalhos de literatura demonstram a ocorrência de resistência cruzada ${ }^{15}$, o que também pode ser observado no presente estudo. Esse resultado era esperado, já que o mecanismo de ação dessas fluoroquinol onas é o mesmo do apresentado pela ciprofloxacina. Porém, duas fluoroquinol onas que ainda se encontram em fase de pesquisa, a clinafloxacina e o WIN-57.273, demonstraram excelente atividade contra amostras resistentes à ciprofloxacina. Pouco ainda se sabe sobre a toxicidade desses compostos, e mais estudos são necessários para uma melhor avaliação do papel clínico dessas fluoroquinol onas. Além disso, os MICS desses compostos foram 8 a 64 vezes mais elevados em amostras resistentes à ciprofloxacina que em amostras sensíveis a esse antimicrobiano, sugerindo que, quando lançadas na prática clínica, esses MIcs possam se el evar ainda mais, inviabilizando o uso clínico desses compostos.

O perfil de sensibilidade de amostras clínicas a antimicrobianos sofre influência importante na manei ra como os antimi crobianos são utilizados em um determinado hospital ou região geográfica. Dessa maneira, é importante que diferentes países, diferentes regiões de um país e mesmo diferentes hospitais de uma região façam aval iações regulares 


\begin{tabular}{|c|c|c|c|c|c|c|c|c|c|c|}
\hline Amostra & Ciprofloxacina & E4695 & E4868 & Fleroxacina & Lomefloxacina & Grepafloxacina & Ofloxacina & Temafloxacina & WIN-57273 & Clinafloxacina \\
\hline 1 & 0,25 & 0,03 & 0,03 & 0,25 & 0,5 & $\leq 0,03$ & 0,25 & $\leq 0.06$ & $\leq 0,12$ & $\leq 0,03$ \\
\hline 2 & 0,12 & 0,03 & 0,015 & 0,25 & 0,5 & $\leq 0,03$ & 0,25 & $\leq 0,06$ & $\leq 0,12$ & $\leq 0,03$ \\
\hline 3 & 0,25 & 0,06 & 0,03 & 0,5 & 0,5 & $\leq 0,03$ & 0,25 & $\leq 0,06$ & $\leq 0,12$ & $\leq 0,03$ \\
\hline 4 & 0,25 & 0,03 & 0,03 & 0,25 & 0,5 & $\leq 0,03$ & 0,25 & $\leq 0,06$ & $\leq 0,12$ & $\leq 0,03$ \\
\hline 5 & 0,25 & 0,03 & 0,03 & 0,25 & 0,5 & $\leq 0,03$ & 0,25 & $\leq 0,06$ & $\leq 0,12$ & $\leq 0,03$ \\
\hline 6 & 8 & 8 & 8 & $>8$ & $>8$ & $>4$ & 8 & 4 & 0,25 & 0,25 \\
\hline 7 & 8 & $>8$ & 8 & $>8$ & $>8$ & $>4$ & 8 & 4 & 0,25 & 0,25 \\
\hline 8 & 8 & 8 & 8 & $>8$ & $>8$ & $>4$ & 8 & 4 & 0,25 & 0,25 \\
\hline 9 & 8 & 8 & 8 & $>8$ & $>8$ & $>4$ & 8 & 4 & 0,25 & 0,25 \\
\hline 10 & 8 & 8 & 8 & $>8$ & $>8$ & $>4$ & 8 & 4 & 0,25 & 0,25 \\
\hline 11 & 8 & 8 & 8 & $>8$ & $>8$ & $>4$ & 8 & 4 & 0,25 & 0,25 \\
\hline 12 & 8 & 8 & 8 & $>8$ & $>8$ & $>4$ & $>8$ & 8 & 0,25 & 0,25 \\
\hline 13 & 8 & $>8$ & $>8$ & $>8$ & $>8$ & $>4$ & $>8$ & 4 & 0,25 & 0,25 \\
\hline 14 & 8 & $>8$ & 8 & $>8$ & $>8$ & $>4$ & 8 & 4 & 0,25 & 0,5 \\
\hline 15 & 8 & $>8$ & $>8$ & $>8$ & $>8$ & $>4$ & 8 & 4 & 0,25 & 0,25 \\
\hline 16 & 8 & $>8$ & $>8$ & $>8$ & $>8$ & $>4$ & 8 & 4 & 0,25 & 0,25 \\
\hline 17 & 8 & 8 & 4 & $>8$ & $>8$ & $>4$ & $>8$ & 4 & $\leq 0,12$ & 0,25 \\
\hline 18 & 8 & 8 & 8 & $>8$ & $>8$ & $>4$ & 8 & 8 & 0,25 & 0,25 \\
\hline 19 & $>4$ & $>8$ & $>8$ & $>8$ & $>8$ & $>4$ & $>8$ & $>8$ & 0,5 & 1 \\
\hline 20 & $>4$ & $>8$ & $>8$ & $>8$ & $>8$ & $>4$ & $>8$ & $>8$ & 0,5 & 1 \\
\hline 21 & $>4$ & $>8$ & $>8$ & $>8$ & $>8$ & $>4$ & $>8$ & $>8$ & 0,25 & 1 \\
\hline 22 & $>4$ & $>8$ & $>8$ & $>8$ & $>8$ & $>4$ & $>8$ & $>8$ & 0,25 & 1 \\
\hline 23 & $>4$ & $>8$ & $>8$ & $>8$ & $>8$ & $>4$ & $>8$ & $>8$ & 0,25 & 0,12 \\
\hline
\end{tabular}

do perfil de sensibilidade de amostras clínicas, pois esses dados podem ser muito úteis na orientação da terapêutica empírica e na avaliação de novos antimicrobianos a serem introduzidos no mercado.

Os resultados do presente estudo mostraram que existe uma diferença importante entre amostra de OSSA e ORSA com relação ao perfil de sensibilidade, e que restam poucas opções para o tratamento de infecções graves causadas por ORSA.

\section{SUMMARY}

In vitro antimicrobial susceptibility of 117 clinical isolates of Staphylococcus aureus from 12 hospitals

Obj ective. To eval uate the anti mi crobial susceptibility pattern of oxacillin susceptible (OSSA) and resistant Staphylococcus aureus (ORSA) isolates to other antimicrobial agents that can be used for the treatment of staphylococcal infections.

Material AND Method. Weevaluated $117 \mathrm{clinical}$ S. aureus isolates from several São Paulo hospitals. Clinical isolates from Campinas, SP and from J oão Pessoa, PB, were also included. The in vitro susceptibility testing was performed by broth microdilution as described by the National Committee for Clinical Laboratory Standards (NCCLS). The minimum inhibitory concentration (MIC) was evaluated for 24 antimicrobial agents, including $\beta$-lactams, fluoroquinolones, aminoglycosides, glycopeptides, macrolides, lincosamides and streptogramins. B oth commercially available and experimental drugs were included in the study. Cross-resistance among fluoroquinolones was evaluated by suscepti bility testing 24 isol ates to 10 fluoroquinol ones.

RESULTS. The antimicrobial agents that showed the highest in vitro acti vity were the gl ycopeptides, the streptogramin RP-59.500, and the mupirocin (100\% susceptibility). Eighty-seven percent of the OSSA and only $38 \%$ of the ORSA isolates were susceptible to ciprofloxacin ( MIC $_{50} 0.25 \mu \mathrm{g} / \mathrm{mL}$ and $>$ $4 \mu \mathrm{g} / \mathrm{mL}$, respectively). Cross-resistance among fluoroquinolones were noted even for the experimental drugs. Two fluoroquinolones remained active against ciprofloxacin-resistant isolates, clinafloxacin and WIN-57.273. However, the ciprofloxacin-resistant isolates had MICS eight-to 64-fold higher than the ciprofloxacin-susceptible isolates, suggesting that the MICS may continue to increase when these fluor oquinol ones become commercially available.

Conclusion. Our results showed a high rate of antimi crobial resistance among $\mathrm{S}$. aureus from the Brazilian hospitals. Very few drugs can still be used for the treatment of staphylococcal infections. [Rev Ass Med Brasil 1997; 43(3): 199-204.]

KEY WORDS: Staphyl ococcus aureus. In vitro antimicrobial activity. Antimicrobial resistance. Fluoroquinolones. Glycopeptides 


\section{REFERÊNCIAS BIBLIOGRÁFICAS}

1. Chambers HF. Methicillin-resistant staphylococci. Clin Microbiol Rev 1988; 1: 173-83.

2. Schwalbe RS, Stapleton JT, Gilligan PH. Emergence of vancomycin resistance in coagulase-negativestaphylococci. N Engl J Med 1987; 316: 927-31.

3. Herwaldt L, Boyken L, Pfaller M. In vitro selection of resistance to vancomycin in bloodstream isolates of Staphylococcus haemolyticus and Staphyl ococcus epidermidis. Eur J Clin Microbiol Infect Dis 1991; 10: 1.007-12.

4. Willey BM, Kreiswirth $B N$, Simor $A E$ et al. Detection of vancomycin resistance in Enterococcus species. J Clin Microbiol 1992; 30: 1.621-4.

5. Daum RS, Gupta S, Sabbagh R, Milewski WM. Characterization of Staphylococcus aureus isolates with decreased susceptibility to vancomycin and teicoplanin: I solation and purification of a constitutively produced protein associated with decreased susceptibility. J I nfect Dis 1992; 166: 1.06672.

6. National Commitee for Clinical Laboratory Standards. Approved standard, M7-A3: methods for dilution antimicrobial susceptibility tests for bacteria that grow aerobically. 3rd ed. Villanova, PA: NCCLS, 1993.

7. Sader HS, Pignatari AC, Hollis RJ, Leme I, J ones RN. Oxacillin and quinolone resistant Staphylococcus aureus in São Paulo, Brazil: a multicenter molecular epidemiology study. Infect Control Hosp Epidemiol 1993; 14: 260-4.

8. Finch RG, Hawley PM, Towner KJ , eds. RP-59500, a semisyn- thetic injectable streptogramin antibiotic. J Antimicrob Chemother 1992; 30(Suppl A): 1-131.

9. Maskell J P, Sefton AM, Williams J D. Comparative in vitro activity of azithromycin and erythromycin against gram-positive cocci, Haemophylus influenzae, and anaerobes. I Antimicrob Chemother 1990; 25(Suppl A): 19-24.

10. Hardy D, Hensey D, Beyer J et al. Comparative in vitro activities of new 14-, 15- and 16-membered macrolides. Antimi crob Agents Chemother 1988; 32: 1.710-9.

11. Pappa KA. The clinical development of mupirocin. J Amer Acad Dermatol 1990; 22: 873-8.

12. Doebbeling $\mathrm{BN}$, Breneman LD, Neu CH et al. Elimination of Staphyl ococcus aureus nasal carriage in health care workers: analysis of six clinical trial with calcium mupirocin ointment. Clin Infect Dis 1993; 17: 466-74.

13. Peterson LR, Quick J N, J ensen B et al. Emergence of ciprofloxacin resistance in nosocomial methicillin-resistant Staphylococcus aureus isolates. Arch Intern Med 1990; 150: 2.151-5.

14. Trucksis M, Wolfson J S, Hooper DC. A novel locus conferring fluoroquinolone resistance in Staphylococcus aureus. J Bacteriol 1991; 173: 5.854-60

15. Piddock LJ V. New quinolones and gram-positive bacteria. Antimicrob Agents Chemother 1994; 38: 163-9.

16. Maple PAC, Hamilton-Miller J MT, Brumfitt W. Differing activities of quinolones against ciprofloxacin-susceptible and ciprofloxacin-resistant, methicillin-resistant StaphyIococcus aureus. Antimicrob Agents Chemother 1991; 35: 345-50. 